\title{
Table of legislation
}

Treaties, Conventions and other Income Tax Assessment Act 1936 ... 2,

International Instruments

Australian-New Zealand Double

Taxation Agreement

(2010)

Art 13(5)

Art 13(7)

Council Directive (EU) 2016

Art 5

First Protocol to the India-Singapore

Tax Treaty of 1994

Art 3 226

Hong Kong-New Zealand Double

Taxation Agreement (2011)

Art 2(2) 103

India-France Double Taxation

Agreement (1992)

India-Mauritius Double Taxation Agreement (1982) 231

Art 1

Art 2 224-7

Art 3 .226

Art 5 226 226

Singapore-India Double Taxation Agreement (1994)

Art 6 226

\section{National Legislation}

\section{Australia}

Double Taxation Relief (Australia)

$$
\text { Order } 1972
$$$$
226
$$

Double Taxation Relief (Australia)

Order 1995

5

Incon

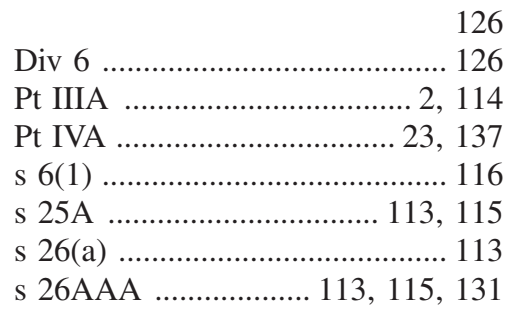

Tax Assessment Act

1997

$116,125,138$

Div 40 124

Div 114 126,129

Div 115 125,127

Div 121 138

Div 125 137

Div 130 118

Div 134

Div 149 118

Div 152 126,138

Div 855 4, 128

Pt 3.1 Pt 3.3 s $6-5$

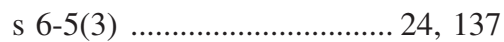

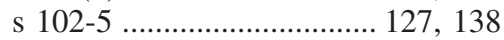

s $102-10$....................................... 138

s $102-15$.............................. 18, 132

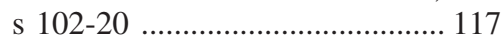

s $103-20$....................................... 130

s $104-10$.......................... 117, 126

s $104-10(1)$................................... 117

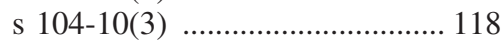

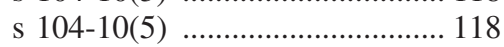

s 104-10(6) .................................. 118

s $104-15$....................................... 117

s 104-20 .......................................... 118

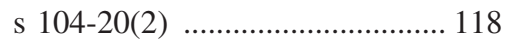

s 104-20(3) ……........................ 118 


\begin{tabular}{|c|c|}
\hline 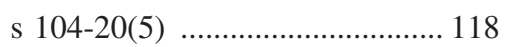 & 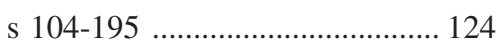 \\
\hline 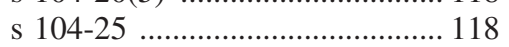 & 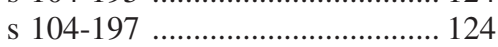 \\
\hline s $104-25(1)$ & 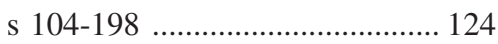 \\
\hline s $104-25(2)$ & s $104-230 \ldots \ldots . .25-6,124,126,138$ \\
\hline s $104-25(3)$ & s $104-230(6)$ \\
\hline 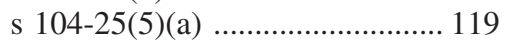 & 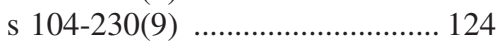 \\
\hline 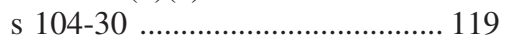 & 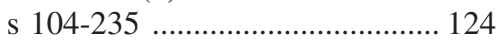 \\
\hline 104-35 ….................. 119, 121 & 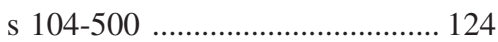 \\
\hline s $104-35(1)$ & 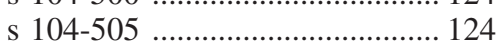 \\
\hline 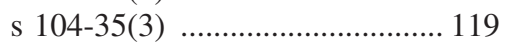 & 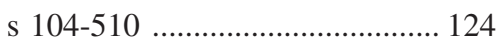 \\
\hline $104-35(5)(\mathrm{c})$ & 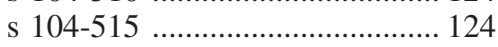 \\
\hline 104-35(5)(d) ................................ 119 & s 104-520 „................................. 124 \\
\hline $104-40$ & s $104-525 \ldots .$. \\
\hline 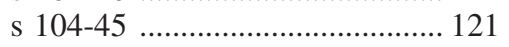 & s $104-535 \ldots$. \\
\hline 104-47 ……....................... 121 & ............. 126, 132 \\
\hline 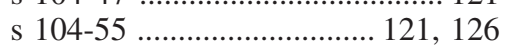 & s $108-5$ \\
\hline 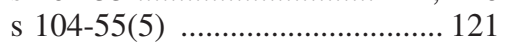 & s $108-5(2)$ \\
\hline $104-60$ & 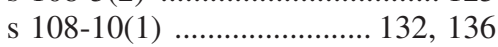 \\
\hline $104-65$............................... 121, 126 & s 108-10(2) ..................................... 136 \\
\hline 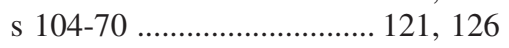 & s $108-10(4) \ldots$ \\
\hline s $104-70(1)$ & 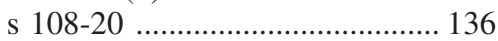 \\
\hline 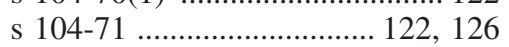 & s $108-20(2)$ \\
\hline 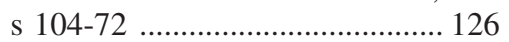 & s $108-20(4) \ldots$ \\
\hline 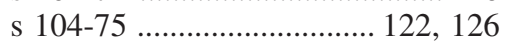 & s $108-55 \ldots \ldots \ldots \ldots$. \\
\hline $104-75(5)$ & s $108-60 \ldots \ldots \ldots . . . .$. \\
\hline 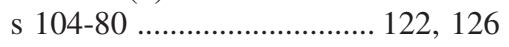 & s $108-70$. \\
\hline 104-85 ….................... 122, 126 & s $108-80 \ldots \ldots \ldots . . . .$. \\
\hline 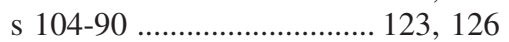 & 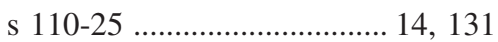 \\
\hline $104-95$ & 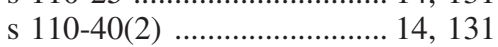 \\
\hline 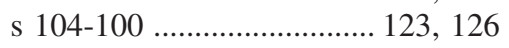 & 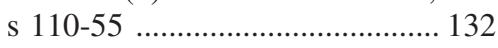 \\
\hline 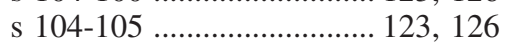 & ....... 11, $132-3$ \\
\hline 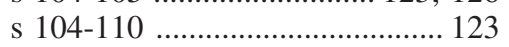 & s $112-30$ \\
\hline 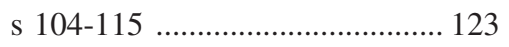 & 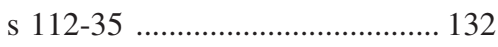 \\
\hline 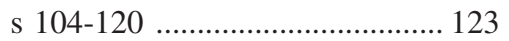 & 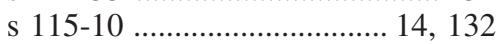 \\
\hline 104-125 & s $115-10(\mathrm{c})$ \\
\hline $104-130$ & s $115-20$ \\
\hline 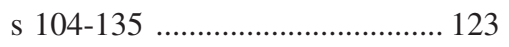 & 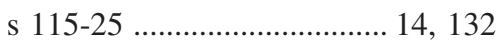 \\
\hline 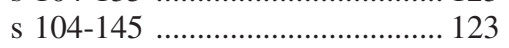 & s $115-25(3)(a) \ldots \ldots \ldots \ldots \ldots \ldots \ldots \ldots . . .4,115$ \\
\hline s $104-150$ & s $115-40$ \\
\hline 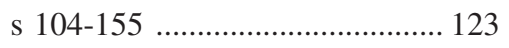 & s $115-100 \ldots \ldots \ldots \ldots \ldots \ldots \ldots \ldots . . .4,14,132$ \\
\hline 104-160 & s $115-100(b)$ \\
\hline 104-165 & s $115-215$ \\
\hline $104-170$ & s $115-280$ \\
\hline 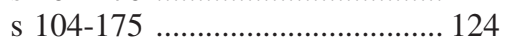 & s $115-290$ \\
\hline 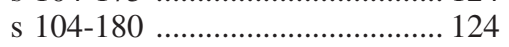 & s $116-20$ \\
\hline 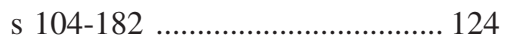 & s $116-30 \ldots \ldots \ldots \ldots \ldots \ldots . .17,130,133$ \\
\hline 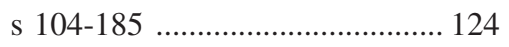 & s 116-30(1) ................................... 130 \\
\hline 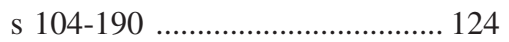 & 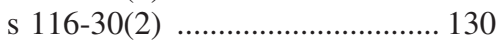 \\
\hline
\end{tabular}




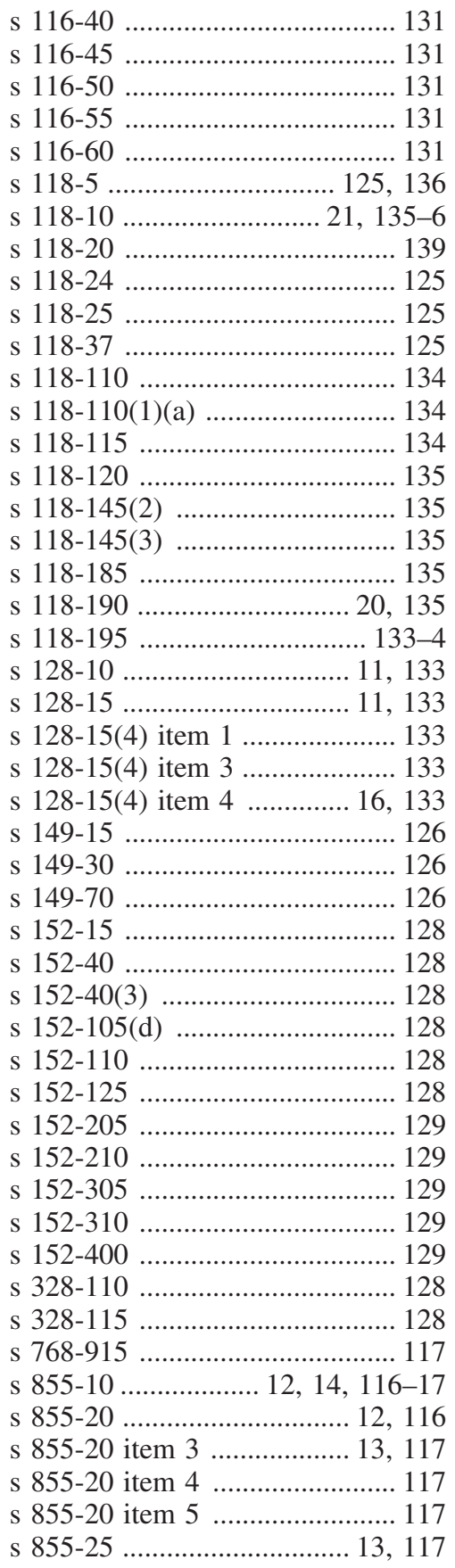

s $855-30 \ldots \ldots \ldots \ldots \ldots \ldots \ldots \ldots \ldots . . . .13,117$

s $855-50$..................................... 133

s 995-1 ................................ 116-17

Sub-div 115-C .................... 14, 132

Sub-div 115-D .................... 14, 132

Sub-div 118-B ..................... 19, 134

Sub-div 124-B ............................ 136

Sub-div 124-C ............................. 137

Sub-div 124-D ............................. 137

Sub-div 124-E ............................. 137

Sub-div 124-F ............................. 137

Sub-div 124-G .......................... 137

Sub-div 124-H ............................ 137

Sub-div 124-I .............................. 137

Sub-div 124-J ............................... 137

Sub-div 124-M ............................ 137

Sub-div 124-N .................. 124, 137

Sub-div 126-A .............................. 136

Sub-div 126-B ............................. 136

Sub-div 126-C ............................... 136

Sub-div 149-C ............................ 126

Sub-div 152-B .................. 122, 128

Sub-div 152-C ................... 122, 128

Sub-div 152-D ............................ 129

Sub-div 152-E .................. 124, 129

Sub-div 768-R .......................... 116

Sub-div 960-M ............................ 129

Income Tax Assessment Amendment

(Capital Gains) Bill 1986 ..... 114

International Tax Agreements Act 1953 .......................................... 133

Migration Act 1958 ....................... 117

Social Security Act 1991 .............. 117

Ch 2, E16 ...................................... 116

Tax Laws Amendment (2006

Measures No 4) Bill 2006 ...... 87

para 4.5 ....................................... 88

para 4.6 ....................................... 88

Taxation Laws Amendment Act (No 4) 1992 .......................... 119

Taxation Laws Amendment Bill

(No 4) 1992 ........................... 120

\section{Canada}

Income Tax Act 1948 ............ 141, 143

c 52 ........................................ 141

s $127(1)(\mathrm{e})$................................. 143 
Income Tax Act 1970-71-72, c 63 s 1 s $117(1)(\mathrm{m})$ 2, 4, 141 Income Tax Act, RSC 1952, c 148 s 32(1)(q) Income Tax Act, RSC 1985 , c $1\left(5^{\text {th }}\right.$ Supp) .......... 152-4, 156-62, 165 art 2 para $121(\mathrm{~g})$

Pt XIII

s 2(1)

s 2(3)

s 2(3)(c)

s 3(a)

s 3(b)

s 3(b)(i)(B)

s $3(d)$

s 3.1

s 38

s 38(a)

s 38(a.1)

s 38(a.2)

s 38(b)

s 39(1)(a)

s 39(1)(b)

s 39(1)(c)

s 39(4)

s $39(5)$

s 39(6)

s 40(1)(a)

s 40(1)(a)(i)

s 40(1)(a)(ii)

s 40(1)(a)(iii)

s 40(1)(b)

s 40(2)(b)

s 40(2)(f)

s 40(2)(g)(i)

s 40(2)(g)(iii)

s 40(3.3)

s 40(3.4)

s 40(3.5)

s 41(1)

s 41(2)(a)

s 41(2)(b)

s 44(1)

s 44.1

s 45(1)(a)

s 45(1)(b)
151

151

... 12

143

154

154-5

154

155

152

156

164

156

166

.. 14

155,159

162

162

156,159

153,159

153,159

156

153

153

153

159

158

158

158

158-9

19-20, 162

162

161

164

161

161

161

164

164

18,164

21,160

21,161

165

165 s $45(1)(\mathrm{c})$

165

s 46(1)

s $46(3)$

21,164

s $46(5)$

164

164

s 53

158

s 53(1)(f) ................................... 161

s 54 ........... 153, 158-9, 161-2, 164

s 55(2) .................... 21-2, 161, 165

s 55(2.1)

21

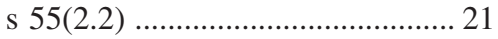

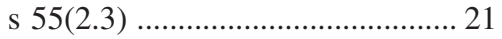

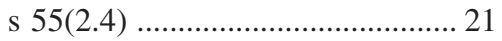

s $55(2.5) \ldots \ldots \ldots \ldots \ldots \ldots \ldots \ldots \ldots \ldots \ldots \ldots . . .21$

s 55(3) ....................... 21, 161, 165

s $55(3.01) \ldots \ldots \ldots \ldots \ldots \ldots \ldots \ldots \ldots \ldots \ldots \ldots . . .21$

s $55(3.02)$.................................... 21

s $55(3.1) \ldots \ldots \ldots \ldots \ldots \ldots . . . . . .21,161,165$

s $55(3.2) \ldots \ldots \ldots \ldots \ldots \ldots . . . . . .21,161,165$

s $55(3.3) \ldots \ldots \ldots \ldots \ldots \ldots \ldots . .21,161,165$

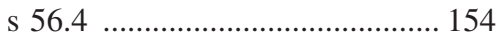

s $69(1)(\mathrm{b})(\mathrm{ii})$.................. 11, 17, 160

s $69(1)(c)$...................... 11, 17, 160

s 70(5)(a) ..................... 11, 16, 160

s 70(5)(b) ................................. 160

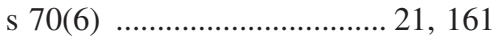

s $70(9)$........................................ 161

s $70(9.31)$.................................... 161

s 73(1) ................................ 21, 161

s 73(1.01) ..................................... 161

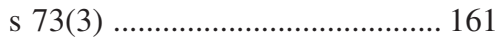

s 73(4) ......................................... 161

s 73(4.1) ….................................. 161

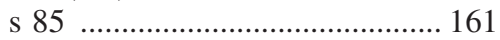

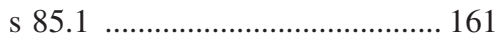

s 86 ....................................... 161

s 87 ................................... 21, 161

s 88 ................................ 21, 161

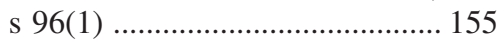

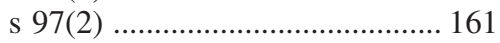

s $98(3) \ldots \ldots \ldots \ldots \ldots \ldots \ldots \ldots \ldots \ldots . . .21,161$

s 98(4) …........................... 21, 161

s $98(5) \ldots \ldots \ldots \ldots \ldots \ldots \ldots \ldots \ldots \ldots . . . . .21,161$

s 104(2) ....................................... 154

s 104(4) ....................................... 160

s 104(14) ..................................... 154

s 104(18) ....................................... 154

s 104(21) .................................... 154

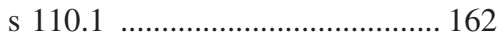




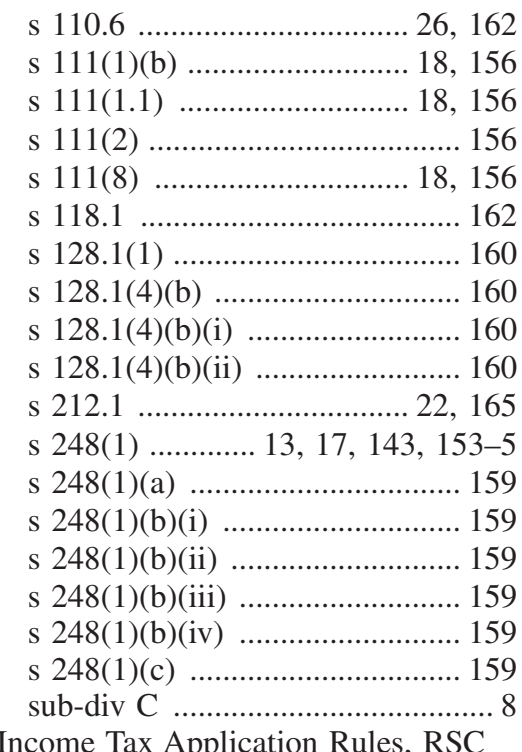

Income Tax Application Rules, RSC 1985 , c 2 ( $5^{\text {th }}$ Supp)

\section{s 26(3)} 159

Income War Tax Act 1917 .... 141, 143 c 28 141

s $3(1)$ 141

s $3(1)(f)$ 143

\section{China}

Enterprise Income Tax Law 2007 .... 6, $13,26,171-3,175,182-4,188$, 190-2, 195

Art 2, para 2 171

Art 2, para 3 171

Art 3 171

Art 4 175

Art 4, para 2 175

Art 6(3)

Art 27 9, 173, 194

Art 28 $175-6$

Art 47 175 190

Implementing Regulations of the Enterprise Income Tax Law 2007 .... 6, 171, 173, 175-6, 195 Art 3, para 1 171 Art 3, para 2 171 Art 4 171 Art 5
Art 6 ........................................... 171

Art 7 ............................................. 171

Art 7(3) .................................... 177

Art 8 ........................................... 171

Art 16 .......................................... 173

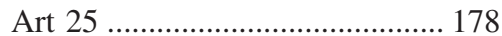

Art 27 ...................................... 175

Art 74 .......................................... 177

Art 75 .......................................... 178

Art 86 ....................................... 175

Art 87 ..................................... 175

Art 88 ........................................ 175

Art 89 ......................................... 175

Art 90 ................................ 175-6

Art 91 .......................................... 175

Art 92 ........................................ 175

Art 93 ......................................... 175

Implementation Regulations of the Individual Income Tax Law 1994 ................................................ 171

Art 2 ............................ 11-12, 171

Art 3 ........................... 11-12, 171

Art 8(9) ....................................... 173

Art 19 ...................................... 176

Art 20 ........................................ 176

Art 22 ....................................... 176

Individual Income Tax Law 1980 .... 6, $26,170,172-3,176-7$

Art 1, para 1 ................. 11-12, 171

Art 1, para 2 ...................... 11, 171

Art 2(9) ...................... 9, 173, 194

Art 3(5) .................................... 175

Art 4 ......................................... 176

Art 5 .............................................. 176

Art 6, para 1(5) ......................... 176

\section{India}

Civil Procedure Code O 30 r 9 ......205

Constitution ..................................... 214

Art 246 ....................................... 214

Art 246(3) .................................. 214

List I(82) of sch 7 .................... 214

List II(46) of sch 7 ................... 214

Direct Taxes Code Bill 2009 .... 233-4

Ch X .................................. 6, 233

Estate (Amendment) Act 1985 ..... 199

Estate Duty Act 1953 .................... 199 


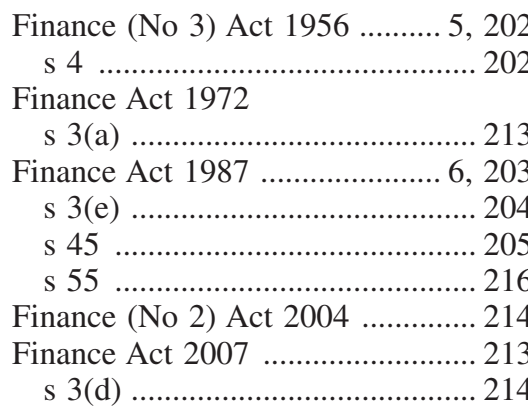

Finance Act 2012 .... 216, 221, 229-30

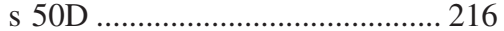
s 112(1)(c) ………………....... 218

Finance Act 2013

s 31 ........................................... 220

Finance (No 2) Act 2014 .............. 223

Finance Act 2015

Explanation 7(i) ......................... 232

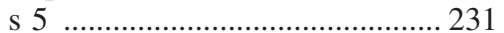

s 13 ........................................... 232

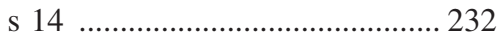

s 76 ............................................ 232

Finance Act 2017 ......... 212, 218, 232

Financial Services Act 2007 ......... 225

s 71 .......................................... 225

Income-tax Act 1922 ................ 5, 196

s $2(4 \mathrm{~A})$....................................... 197

s 12B ............................ 197, 201-2

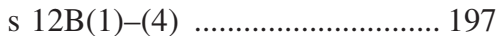

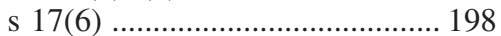

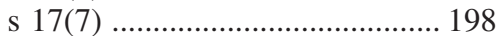

Income-tax Act 1961 ... 196, 202, 205,

209, 212, 215, 219

Ch X-A

227

Ch XX-A

217

Ch XX-C

217

s $2(14)$

s 2(24) 212-13, 223, 229

s 2(24)(vi)

212

s 2(47)

212, 214, 229

s 2(47)(i)-(iv)

215

s 9(1)

231

s 9(1)(i)

s 10 (1A)

229

s 10(34A)

s $10(37)$

s 10(37A) s 10(38)

15,209

s 45

s 45(1)

5, 212

s 45(2)

212

s 45(3)

s 45(4)

215

s 47

215

s 47(iii)

215

s 47(iv)

215

s $47(\mathrm{v})$

215

s 47(via)

215

s 47(vic)

s 48

s 50

215

216

216

s $50 \mathrm{C}$

s 50D …...................................... 216

s 54 ...................................... 221-2

s 54(1) ....................................... 222

s 54B ……………………... 221-2

s 54D …................................ 221-2

s 54EA ……............................. 222

s 54EB …….............................. 222

s 54EC ……......................... 221-2

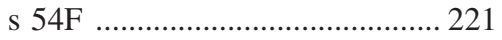

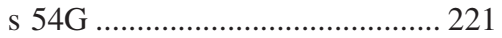

s 54GB ……………………... 221

s 55(2)(aa) …………………..... 216

s 56(2)(vii) ................................ 215

s 56(2)(vii)(a) ........................... 215

s $70(2)$........................................ 219

s $70(3)$......................................... 219

s 71(3) .......................................... 219

s 74(1)(a) .................................. 219

s 74(1)(b) ................................. 219

s 74(2) ........................................ 219

s 90(2) .......................................... 219

s $111 \mathrm{~A}$....................................... 206

s 112 ......................... 206, 208, 218

s 112(1)(c) .............................. 218

s 115AD ............................ 13, 219

s 115QA ...................................... 220

Income-tax and Business Profits Tax

(Amendment) Act 1948

s 5(a)

197

Taxation Laws (Amendment and Miscellaneous Provisions) Act, 1986 206, 214 s 2 206 


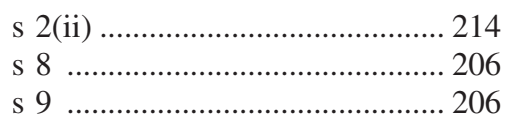

\section{Mauritius}

Mauritius Offshore Business Activities Act 1992 224-5 s $94 \mathrm{~A}$ 226

\section{The Netherlands}

Corporate Income Tax Act 1969 .... 17, 236, 273

Art 6 253

Art $14 \mathrm{c}(1)$ 255

Art $15 \mathrm{c}$ 246

Art 22 $252-3$

Dividend Withholding Tax Act ..... 266 Arts 1-17 266

General Taxes Act Art 31 23

Implementing Decree Personal Income Tax Act [Uitvoeringsbesluit inkomstenbelasting] 2001 ..... 257 Implementing Rules Recovery Act [Uitvoeringsregeling Invorderingswet] 1990

Art 2 255

Art 3 256

Art 3a 256

Art 6d 246

Income Tax Decree 1941 240

Inheritance and Gift Tax Act 1956 236,273

Art 1 . 11

Personal Income Tax Act 1914 $239-40$

Personal Income Tax Act 1964 ...... 65, 240-1, 252

Personal Income Tax Act 2001 ...... 17, 65, 236, 240-1, 260-1, 266, 273,276

Art 2.3 241

Art 2.4 241

Art 2.8 255

Art 2.9 255 Art 2.10 .. 13, 241, 246, 248-9
Art 2.10(2) 20,249

Art 2.10a ........ 13, 241, 246, 248-9

Art 2.11a .................................. 252

Art 2.12 ................................... 250

Art 2.13 .................................... 264

Art 2.14 ................................... 241

Art 2.14(3) ............................... 241

Art 3.1 ....................................... 242

Art 3.110-3.123a ...................... 249

Art 3.123a ................... 242, 249-50

Art 3.124-3.143 ….................... 242

Art 3.25-3.30a ........................... 242

Art 3.54 ................................... 243

Art 3.55-3.59 …......................... 244

Art 3.60 ................................... 246

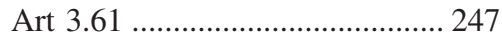

Art 3.62-3.65 ............................ 244

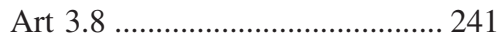

Art 3.90-3.99a .......................... 248

Art 4.2-4.11 ............................... 251

Art 4.12(a) ................................ 250

Art 4.12(b) ................................ 250

Art 4.13 ....................................... 253

Art 4.14 ...................................... 253

Art 4.16(1)(d) ........................... 254

Art 4.16(1)(e) ............................ 254

Art 4.16(1)(h) ........................... 255

Art 4.17 ...................................... 254

Art 4.17a .................................... 254

Art 4.17b ..................................... 254

Art 4.17c .................................... 254

Art 4.22 ..................................... 254

Art 4.25 ................................... 258

Art 4.39 ..................................... 254

Art 4.39a ................................... 254

Art 4.39b …................................ 254

Art 4.39c ................................... 254

Art 4.41 .................................... 254

Art 4.42 .................................... 255

Art 4.42a .................................... 255

Art $4.43-4.46 \ldots \ldots \ldots \ldots \ldots \ldots \ldots \ldots . . . . . . . . . . . . .253$

Art 4.53 .................................... 252

Art $5.1-5.18 \mathrm{~b} \ldots \ldots \ldots \ldots \ldots \ldots \ldots \ldots \ldots . . . . . . . . . . . . .262$

Art $5.19 \ldots \ldots \ldots \ldots \ldots \ldots \ldots \ldots \ldots . . . . . . . . . . . . . . .262-3$

Art 5.20 ..................................... 262

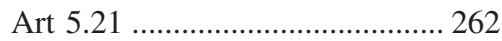

Art 5.22 .................................. 262

Art 5.23 .................................. 262 


\begin{tabular}{|c|c|}
\hline Art $6.1-6.39 a$...................... 242, 251 & 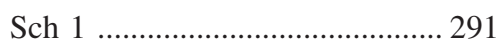 \\
\hline 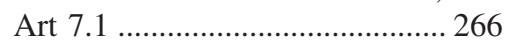 & s BB 1 \\
\hline Art 7.2 & 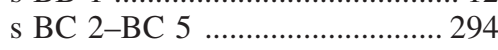 \\
\hline Art $7.2(2)$ (a) .................... 13, 246-7 & s BD $1 \ldots \ldots \ldots \ldots . . . . .$. \\
\hline Art 7.2(2)(b) & s BD $1(4) \ldots \ldots \ldots$. \\
\hline 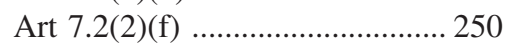 & s BD 1(5). \\
\hline Art $7.2(4)$ & s BD $2 \ldots \ldots \ldots . . .$. \\
\hline Art 7.2(5) & s BD $3 \ldots \ldots \ldots \ldots$ \\
\hline Art 7.2(6) & s BD 3(3) ....... \\
\hline 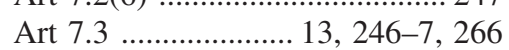 & s BD 4 ................. \\
\hline 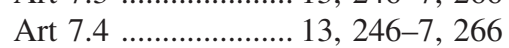 & s BG 1 ....................... \\
\hline 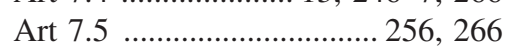 & s CA $1(2) \ldots$ \\
\hline Art 7.5(4) & s CB $1 \ldots .$. \\
\hline Art $7.5(5)$ & s CB $3 \ldots \ldots$ \\
\hline 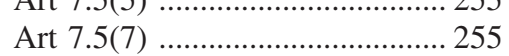 & $\ldots .96,296,299$ \\
\hline Art $7.6 \ldots \ldots \ldots \ldots \ldots \ldots .2 .256,266$ & s CB 5 ................... \\
\hline Art $7.6(3) \ldots 2025$ & s CB 6-CB $13 \ldots \ldots \ldots \ldots \ldots . . . . .93,106$ \\
\hline 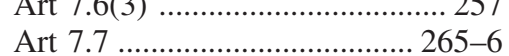 & s CB $14 \ldots \ldots \ldots \ldots \ldots \ldots . . .93,106,298$ \\
\hline Art & s CB 15 .. \\
\hline Art 7.8 & s CB 15B \\
\hline Art $8.8 \ldots \ldots \ldots \ldots \ldots \ldots \ldots \ldots \ldots . .242,248$ & s CB $15 \mathrm{C}$. \\
\hline 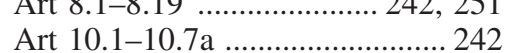 & s CB $16 \mathrm{~A} \ldots \ldots \ldots \ldots$ \\
\hline $\begin{array}{l}\text { Art } 10.1-10.7 \text { a ........................... } 242 \\
\text { ecovery Act [Invorderingswet] }\end{array}$ & s CB $16-C B 18$.. \\
\hline $\begin{array}{l}\text { ecovery Act [Invorder } \\
1990\end{array}$ & s CB 19-CB 23 \\
\hline 1990 & 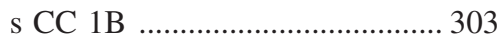 \\
\hline Art 25a …................................... 246 & s CE $9 \ldots$ \\
\hline Art 25(8) ................................... 255 & s CE 10 . \\
\hline Art 25(9) ….................................. 256 & s CQ $5 \ldots \ldots \ldots \ldots \ldots \ldots \ldots \ldots$ \\
\hline 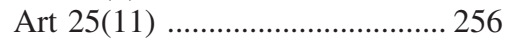 & s CQ $5(1)(d) \ldots \ldots \ldots$. \\
\hline Art $28(3) \ldots \ldots \ldots \ldots . . .$. & s CQ $5(1)(\mathrm{e})$. \\
\hline Art 28(4) …................................ 255-6 & s DA $2(2) \ldots \ldots$ \\
\hline Tages Tax Act [Wet op de & 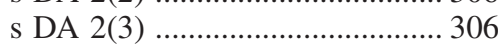 \\
\hline loonbelasting] 1964 & s DB $23 \ldots \ldots \ldots \ldots . . . .$. \\
\hline Art 12a & s DB 26-DB $29 \ldots \ldots \ldots \ldots$ \\
\hline & s EB 2(3) \\
\hline w Zealand & s EW 1(2)(a) ......... \\
\hline dministration Act 1969 & s EW 1(3)(b) ............. \\
\hline s 77 & s EW $3 \ldots \ldots \ldots \ldots \ldots \ldots$ \\
\hline oods and Services Tax Act & s EX 1-EX $28 \ldots .$. \\
\hline 1986 & s EX 44-EX $57 \ldots$. \\
\hline come Tax Act 1976 ..................... 285 & 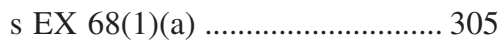 \\
\hline 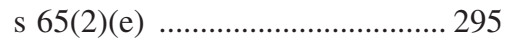 & 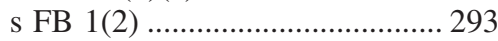 \\
\hline 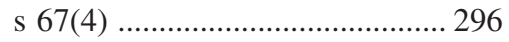 & s FB $2 \ldots \ldots \ldots . . .$. \\
\hline Icome Tax Act 1994 ...................... 285 & s FB 3A \\
\hline Icome Tax Act 2007 ........... 106, 285, & 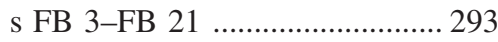 \\
\hline $291,294,305-6$ & s FC 1-FC 8 \\
\hline Pt C & 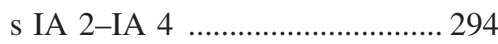 \\
\hline Pt FC 293 & s IQ 1A-IQ 9 \\
\hline
\end{tabular}




$$
\begin{aligned}
& \text { s YA } 1 \\
& \text { s YD } 1 \\
& \text { s YD } 2
\end{aligned}
$$

292-3, 307

291

291

Subpt CB

Subpt EW

Subpt EX

Income Tax Amendment Bill (No 2)

1986

304

Land and Income Assessment Act

1891 285

Land and Income Assessment Act 1900 298

s 59(2) 298

s 59(3)

295

Land and Income Tax Act 1916 295, 298

s $85(\mathrm{c})$ 295

Land and Income Tax Act 1923 ... 298 s 79(c) 295

s 79(1)(c) 296

Land and Income Tax Act 1954 295-6 s $88 \mathrm{~A}$ 295,298

s $88 \mathrm{AA}$ 296

s 88 (c) 295, 297-8

Land and Income Tax Amendment Bill 1973 .................................. 296 s 9 ............................................. 296

Property (Relationships) Act 1976 ........................................ 293

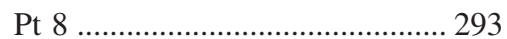

s 21-21C ...................................... 293

s 25 .......................................... 293

Property Speculation Act 1973 ..... 297

Resource Management Act 1991 298

Tax Administration Act 1994 ........ 285 s 33 307 s $33 \mathrm{~A}$ 307

Taxation (Bright-line Test for

Residential Land) Act 2015 .... 93

s GB 52 94,99

s GB 52(1) .. 94

Taxation Review Authorities Act 1994 s 18 299

\section{South Africa}

Estate Duty Act, 45 of 1955 ......... 310

Income Tax Act, 28 of 1914 ............. 4

Income Tax Act, 58 of 1962 ........ 310, $315,325,327,332,334$

Pt III ........................................... 330

Pt V ............................................ 310

Sch 8 .......... 5, 315, 325, 327, 330, 332-3

Sch 8 , para 1 317

Sch 8, para 2(1)(b) ........ 11-13, 316

Sch 8, para 2(2) ............. 11-13, 316

Sch 8, para 5 ............................. 315

Sch 8, para 11 .............. 11, 17, 319

Sch 8, para 11(1)(g) .................. 320

Sch 8, para 12 ............................ 319

Sch 8, para 12A .................. 16, 319

Sch 8, para 13 .............................. 10

Sch 8, para 15 ............................ 324

Sch 8, para 20 ............ 14, 315, 321

Sch 8, para 24 ..................... 25, 317

Sch 8, para 27 ............................. 323

Sch 8, para 30 .................... 25, 322

Sch 8, para 33 ............................. 321

Sch 8, para 35 .................... 14, 315

Sch 8, para 44 ............................ 327

Sch 8, para 46 .............................. 20

Sch 8, para 53(2) ....................... 315

Sch 8, para 54 ........................... 316

Sch 8, para 55 ............................. 316

Sch 8, para 60 ............................ 316

Sch 8, para 62 ........................... 316

Sch 8, para 68-71 ..................... 325

Sch 8, para 80 .................... 18, 325

Sch 8, para 97 .................... 25, 323

Sch 8, Pt II ................................. 18

Sch 8, Pt VII ..................... 20, 315

s 2 ....................................... 326-7

s $22(8)$....................................... 320

s 26A ……………...................... 315

s 80A-80L ………..................... 332

\section{United Kingdom}

Capital Gains Tax Act 1979 .......... 340

Corporation Tax Act 2009

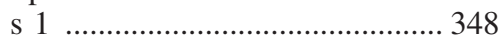

s 5 ………………….......... 348 
s 19

Finance Act 1965

Finance Act 1968

Finance Act 1982

s 86

Finance Act 1988

s 96

Finance Act 1989

s 30

Finance Act 2008

Sch 3

s 6

s 7

Finance Act 2013

s 206

Finance Act 2015

Sch 7

Income Tax Act (Pitt's Act) 1799 ...

Income Tax Act (Addington's Act) 1803

Income Tax Act 1842

Income Tax Act 1918

Income Tax Act 1945

Income Tax Act 2007

$$
\text { s } 714
$$

Income Tax (Trading and Other Income) Act 2005

$$
\text { s } 619
$$

Taxation of Chargeable Gains Act 1992 $7,335,340,348,352$

Ch II

Ch IV

Pt II

Pt IV

s 1

$\mathrm{s} 1(2)$

s 2

s 3(4)

s 8

s 9

s 10

s $10 \mathrm{~A}$

s 10AA(4)

s $10 \mathrm{~B}$

s 11

s 12

s 13

s 14
348

46

339

350

349

339

343

340

343

359

348

... 1

12,25

.. 1-2

143
344

360

360 347

347

13, 350

335

347

$12,18,351$

$12,25,348$

353

353

348

. 12,25

$12,25,348$

$12,25,354$

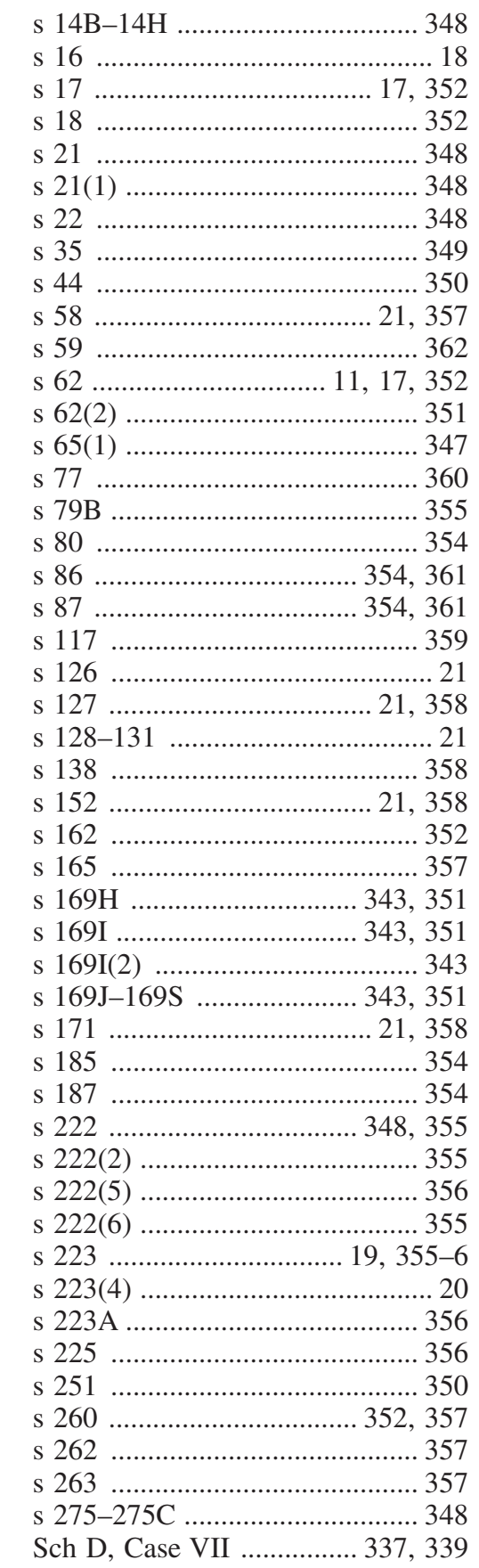

48

52

352

348

348

349

357

352

351

360

355

354

59

21

358

358

358

357

351

343

351

354

354

355

356

5-6

20

356

350

357

357

348

Sch D, Case VII ................ 337, 339 


\section{USA}

American Jobs Creation Act of 2004

§ 804(a)-(c) ............................... 386 § 804(e) ....................................... 386

Constitution

Art I 367

Art I, § 8, cl 1 367

Sixteen Amendment ...... 363, 365-7

Excise Act 365

Foreign Investment in Real Property Tax Act of 1980 373

Health Insurance Portability and Accountability Act of 1996 $\S 511$ 386

Heroes Earnings Assistance and Relief Tax Act of 2008 $\S 301$ (a) 387

Internal Revenue Code 1986 ....... 364, $369,372-4,378,381$

26 CFR § 1.10-1(a) 372

26 CFR $\$ 1.1002-1(b)$ 379

26 CFR $\$ 1.1002-1(d)$ 379

26 CFR § 1.1031(d)-1(e) .......... 378

26 CFR $\S 1.1033(\mathrm{a})-2(\mathrm{c})(2)$...... 380

26 CFR $\S 1.1041-1 \mathrm{~T}$...

26 CFR § 1.1041-1T(d) 380

26 CFR $\$ 1.1233-1(\mathrm{a})(1)$ 380

26 CFR $\$ 1.355-1(\mathrm{~b})$ 396

26 CFR $\S 1.355-2(\mathrm{~b})$ 384

26 CFR $\S 1.355-2$ (c) 384

26 CFR § 1.355-2(d) 384

26 CFR $\$ 1.355-3$ (b)(2)(iv) ...... 384

26 CFR § 1.355-3(b)(3)(ii) ....... 384

26 CFR § 1.368-1(a) .................. 385

26 CFR § 1.368-1(b) ................. 385

26 CFR § 1.368-1(d) ................. 385

26 CFR $\$ 1.368-1(d)(5)$............. 385

26 CFR $\S 1.368-1(\mathrm{e})$.................. 385

26 CFR $\S 1.368-1 \mathrm{~T}(\mathrm{e})(2)(\mathrm{v})$ Ex (1) 385

26 CFR \$ $1.752-6$ 392

26 CFR $\S 1.752-7$ 392

26 USC ... 8

26 USC $\$ 1$ $11-12,372,387$

26 USC $\$ 1(\mathrm{~h})$ $18,372,375-6$

26 USC $\$ 1(\mathrm{~h})(2)$ 14,377

26 USC $\S 1(\mathrm{~h})(5)(\mathrm{A})$
26 USC $\S 1(\mathrm{~h})(6)(\mathrm{A})$ 377

26 USC $\S 1(\mathrm{~h})(9)$ 14, 377

26 USC § 11 ..... 3, 11-12, 372, 387

26 USC $\$ 61$ 363,372

26 USC § 61(a)(3) 366

26 USC $\S 102$ 372

26 USC $\$ 121$ 20, 381

26 USC § 163 20

26 USC $\$ 165$ (a) 378

26 USC $\$ 165(\mathrm{c})$ 378

26 USC $\$ 165(\mathrm{~g})$ 378

26 USC $\S 165(\mathrm{~h})(2)$.................... 378

26 USC § 166(d)(1)(B) ............. 378

26 USC $\S 311$.............................. 382

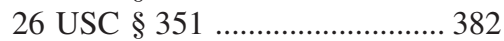

26 USC § 351(b) ....................... 382

26 USC § 351(f) ........................ 382

26 USC $\$ 354$

26 USC $\$ 355$ 21,384

26 USC § 355(a)(1)(B) ............ 384

26 USC $\S 355(\mathrm{a})(1)(\mathrm{C})$.............. 384

26 USC $\S 355(\mathrm{~b})(1)(\mathrm{A})$.............. 384

26 USC $\S 355(\mathrm{~b})(1)(\mathrm{B})$.............. 384

26 USC $\S 355(\mathrm{~b})(2)(\mathrm{D})$............. 384

26 USC § 355(d) ...................... 384

26 USC § 355(e) ....................... 384

26 USC § 356 ................. 21, 383-5

26 USC § 357 ..................... 21, 384

26 USC $\S 357(\mathrm{c}) \ldots \ldots \ldots \ldots \ldots \ldots \ldots . . . . . . . . . . . .383$

26 USC $\S 358$................. 21, 383-4

26 USC $\S 361$................. 21, 384-5

26 USC $\S 362$................. 21, 383-4

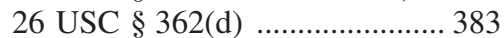

26 USC $\$ 362(\mathrm{e})$......................... 383

26 USC § 362(e)(2) ................... 383

26 USC $\$ 368$...................... 21, 384

26 USC $\S 368$ (a) .................... 384-5

26 USC § 368(a)(1) ..................... 385

26 USC $\S 368(\mathrm{a})(1)(\mathrm{A})$............... 385

26 USC $\S 368(\mathrm{a})(1)(\mathrm{B})$............. 385

26 USC $\S 368(\mathrm{c})$............... 382, 384

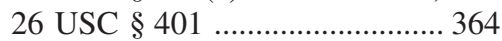

26 USC $\S 408$.............................. 364

26 USC $\S 465$............................. 388

26 USC $\S 469$............................. 388

26 USC $\S 475$....... 10, 28, 364, 374

26 USC $\S 514$............................ 378 


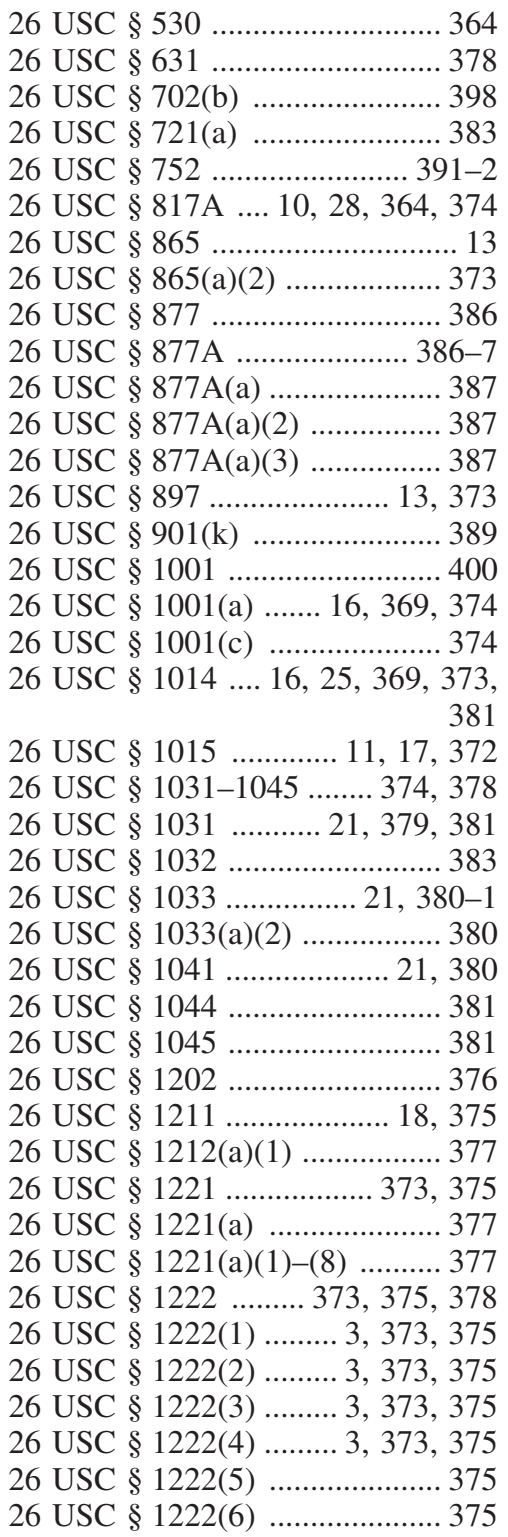

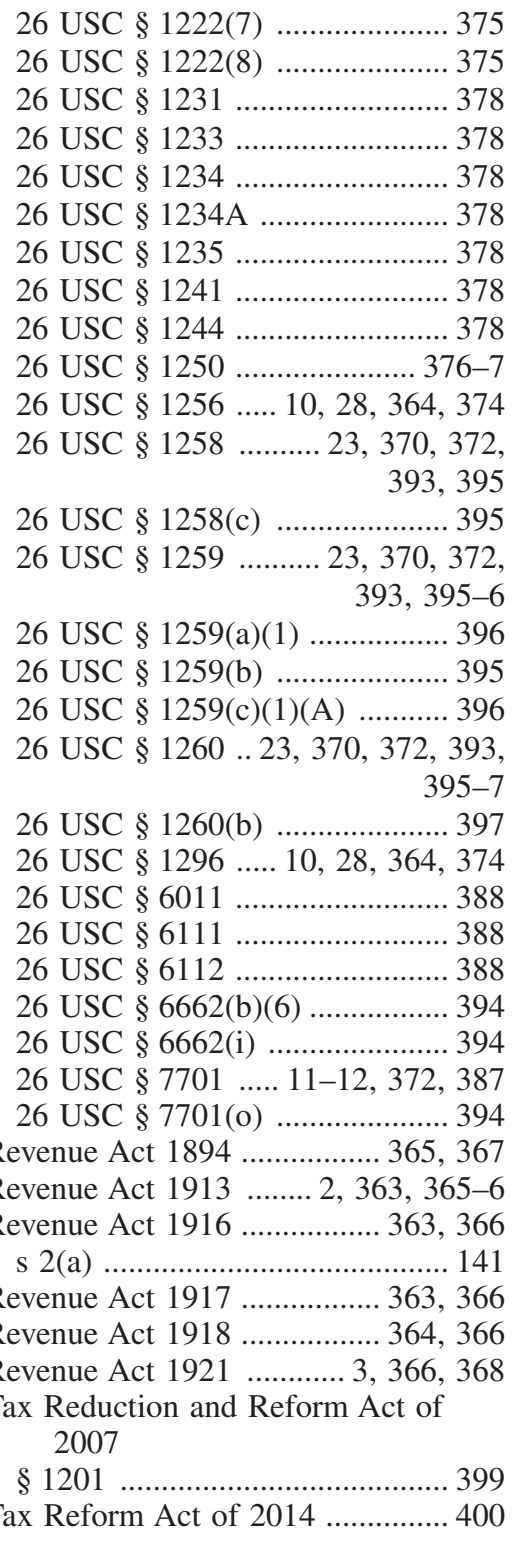


Michael Littlewood and Craig Elliffe - 9781784716028 Downloaded from PubFactory at 04/26/2023 01:29:38AM via free access 